\title{
Predicting leaf state effects on radiowaves based on propagation loss measurements
}

\author{
Mohammed Saleh H. Al Salameh \\ Department of Electrical Engineering, Jordan University of Science and Technology, Irbid 22110, Jordan, salameh@ just.edu.jo
}

\begin{abstract}
A new propagation model is proposed for estimating the attenuation of wireless communication signals in woodland environments. After rainfall or snowfall, the components of the woodland area become moist which degrades the received signal level. To take this into account, the model considers wet/dry states of the foliage, and its dependency on the operating frequency. The parameters of the propagation loss model are optimized using the least squares method. To demonstrate the validity and usefulness of the model, computed results are compared with measured data where excellent matching is observed. It is noted that not only the foliage and rainfall affect the propagation phenomenon, but also wet foliage condition after rainfall contributes to the fading of the wave.
\end{abstract}

\section{Introduction}

Communications in forest environment include battlefield sensor networks and wireless communications as illustrated in Fig. 1, wireless sensor networks for precision agricultural applications, fire and rescue services, private mobile radio systems, and security services. Furthermore, cognitive radio operates at varying frequencies as it adjusts radio-frequency system channel parameters in order to capture the best quality communications. Thus, the need arises for evaluating the performance of the propagation at various frequencies. Propagation of the wave may occur in different environments including the forest that has significant effect on the propagation and attenuation of wireless signals [1], [2]. These considerations lead to the need for accurate modeling and prediction of the path loss through vegetation areas which may exist as well inside cities, in addition to rural areas and forests.

Wave propagation in forest was examined by the use of a dielectric slab model for frequencies 1-100 MHz [3]. The terrain effects of the forest were modeled using the knife edge diffraction model [4]. Curves were derived from measurements along road sections in large forests [5]. An empirical model was presented based on measurements of attenuation in the forests of India [6]. The trees were modeled as a random half-space medium of discrete, lossy scatterers at $11.2 \mathrm{GHz}$ [7]. The distribution function of the fading channel was found to be Rician [8], [9], [10] for cellular communications. The uniform theory of diffraction was applied to calculate the path loss in the presence of lossy trees and buildings for satellite mobile communications [11]. An empirical model was used to represent the propagation loss associated with near ground communications in a tropical forest [12]. Models for forest propagation were compared in order to find a suitable model for the forests of Nigeria [13]. A review is available on rain effects on propagation in addition to the effects of wave polarization [14]. Mostly, analytical models are more accurate than empirical models. However, analytical models are available only for specific geometries and situations. Thus, empirical path loss models based on measurements in forests and vegetation areas are attractive for modelling radio-channel behavior for wireless communication systems and networks. Overall, proper modeling of radio-channel helps in designing cost-effective and suitable communication systems with lower operating power that possibly decreases the risk of undesirable negative effects on the humans and on the ecological system [15].

Besides that, as far as the author knows, there is limited research work done on the effects of weather conditions on the propagation of radiowaves in woodland areas. This paper presents a new optimized propagation loss model involving the effects of wet foliage after rainfall/snowfall and also considers varying frequency of operation. To show the usefulness and validity of the new model, calculated results are compared with measured data in forest areas where agreement was obtained. The least squares technique is used in this paper to obtain the parameters of the optimized model. 


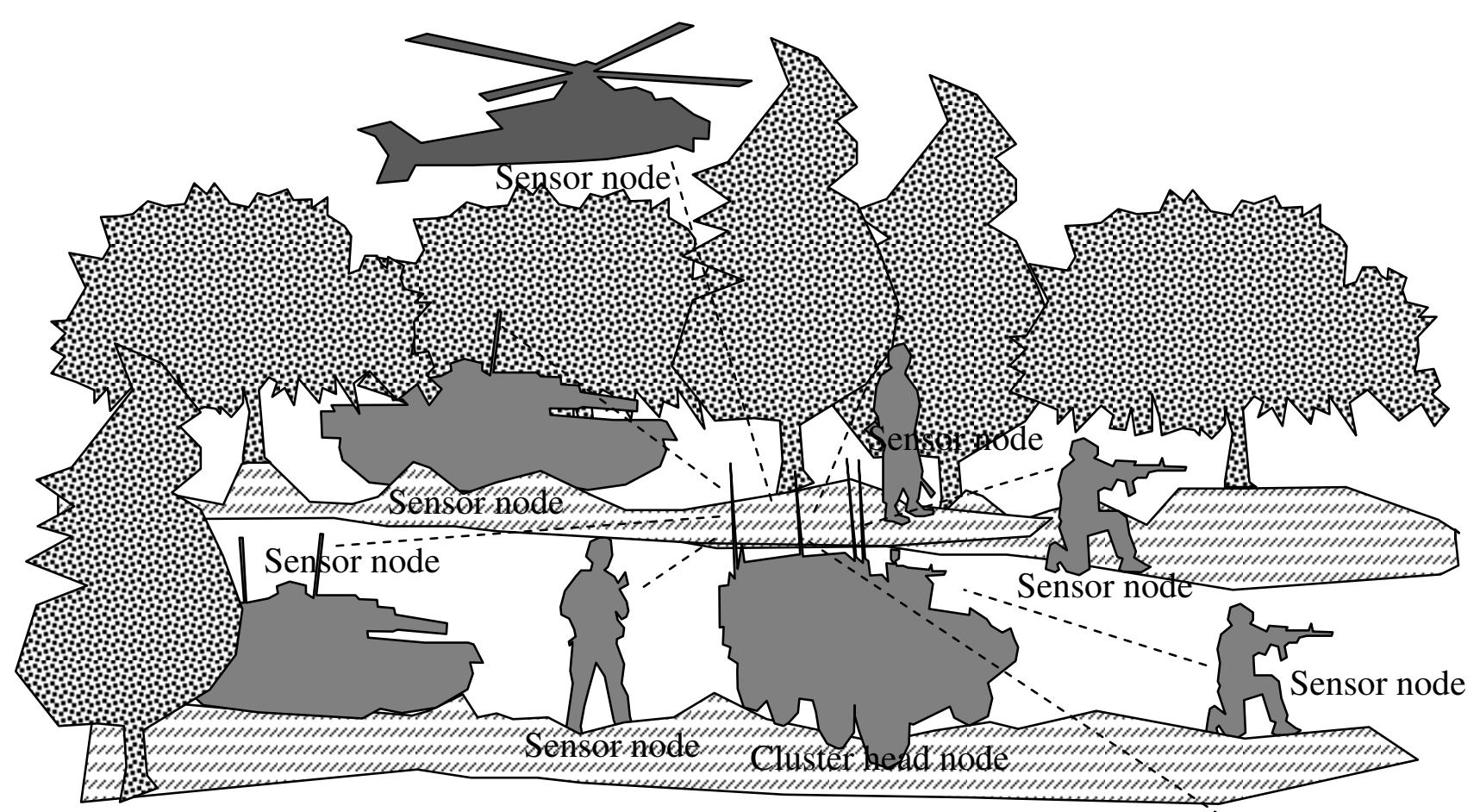

To military base

Fig. 1. Military communication system in woodland, where radiowaves are essential for such system.

\section{Model Optimization}

A scenario of radiowave propagation in forest environment is illustrated in Fig. 1 where infantry soldiers and armored vehicles need to communicate with their command, and the command officers in turn need to communicate with their military base. In woodland areas, the signal from the transmitter to the receiver will suffer significant attenuation and spreading as it passes through the trees in addition to the shrubs and grass on the ground. After rainfall, the area becomes moist, and this condition increases the signal attenuation. Of course, if this is not taken into account during the design of the communication system, the signal will be severely attenuated resulting in possible interruption of the communication link.

The path loss $\mathrm{P}_{\mathrm{L}}$ of a radiofrequency signal between the input of the transmitter and the output of the receiver is [16]:

$$
P_{L}=G_{t}+G_{r}-L-L_{r}-L_{t}
$$

where $L_{t}, L_{r}$ are losses in the transmission lines of the transmitter and receiver, $\mathrm{L}$ is the total propagation loss including vegetation losses, and $G_{t}, G_{r}$ are the antenna gains of the transmitter and receiver.

The general expression [17] for propagation loss will be optimized:

$$
L=A+B \log (d)+C d
$$

Where $L$ is the propagation loss in $\mathrm{dB}, A, B$ and $C$ are parameters to be determined based on the available measurements, and $d$ is the distance in meters between the transmitter and receiver. However, this equation depends only on distance. It is required to find the dependency of the propagation loss on other parameters such as the frequency of operation, condition of the foliage, i.e., wet or dry. Below, the optimum values of these parameters will be determined and augmented to the proposed model.

The values of the parameters $A, B$ and $C$ in equation (2) will be optimized through the application of the least squares technique. To that end, the squared errors (SE) should be minimized:

$$
S E=\sum_{i=1}^{N}\left(P_{i}-M_{i}\right)^{2}
$$

where $M_{i}$ is measured value and $P_{i}$ is the proposed model value of the propagation loss at the points $i$ for a total number of $N$ data points.

Combining equations (2) and (3) yields:

$$
S E=\sum_{i=1}^{N}\left(\left[A+B \log \left(d_{i}\right)+C d_{i}\right]-M_{i}\right)^{2}
$$

To minimize the errors, the partial derivatives of equation (4) with respect to the parameters A, B, C are set to zero, which produces:

$$
N A+\left(\sum_{i=1}^{N} \log \left(d_{i}\right)\right) B+\left(\sum_{i=1}^{N} d_{i}\right) C=\sum_{i=1}^{N} M_{i}
$$




$$
\begin{gathered}
\left(\sum_{i=1}^{N} \log \left(d_{i}\right)\right) A+\left(\sum_{i=1}^{N}\left\{\log \left(d_{i}\right)\right\}^{2}\right) B+ \\
\left(\sum_{i=1}^{N} \mathrm{~d}_{\mathrm{i}} \log \left(d_{i}\right)\right) C=\sum_{i=1}^{N} \mathrm{M}_{\mathrm{i}} \log \left(d_{i}\right) \\
\left(\sum_{i=1}^{N} d_{i}\right) A+\underset{\left.\sum_{i=1}^{N} \mathrm{~d}_{\mathrm{i}} \log \left(d_{i}\right)\right) B+\left(\sum_{i=1}^{N}\left\{d_{i}\right\}^{2}\right) C=}{(7)}
\end{gathered}
$$

Substituting the measured data [18] at $300 \mathrm{MHz}$, into equations (5)-(7), gives the equations:

$$
\begin{gathered}
10 A+24.67 B+4150 C=1144.04 \\
24.67 \mathrm{~A}+62.45 B+11355.88 C=2894.03 \\
4150 A+11355.88 B+2637500 C=525110.5
\end{gathered}
$$

The solution to these equations (8)-(10) can be obtained by iteration techniques or by gauss elimination method: $\mathrm{A}=2.194, \mathrm{~B}=45.6, \mathrm{C}=-0.0007$

Introducing these values into equation (2), an optimized model is achieved:

$$
L_{F}=2.194+45.6 \log (d)-0.0007 d
$$

where the subscript $\mathrm{F}$ is added to the letter $\mathrm{L}$ to indicate forest propagation. The last term in the above equation doesn't significantly contribute to the propagation loss value, and thus may be discarded leaving a linear equation in $\log (\mathrm{d})$ :

$$
L_{F}=2.194+45.6 \log (d)
$$

Since equation (12) has only two parameters, i.e., the third parameter $\mathrm{C}$ in equation (2) turns out to be negligible for our model, we want to optimize equation (2) after removing the parameter $\mathrm{C}$ as follows:

$$
L_{F}=A+B \log (d)
$$

Going along the procedure outlined above to minimize the squared errors, results in two equations rather than three because we now have only two unknowns:

$$
\begin{gathered}
N A+\sum_{i=1}^{N} \log \left(d_{i}\right) B=\sum_{i=1}^{N} M_{i} \\
\sum_{i=1}^{N} \log \left(d_{i}\right) A+\sum_{i=1}^{N}\left\{\log \left(d_{i}\right)\right\}^{2} B=\sum_{i=1}^{N} \mathrm{M}_{\mathrm{i}} \log \left(d_{i}\right)
\end{gathered}
$$

Introducing the measured data at $300 \mathrm{MHz}$ into these equations gives a preliminary optimized model:

$$
L_{F}=3.12+45.11 \log (d)
$$

In order to extract optimized frequency dependence, the measured path losses with foliage for different frequencies are used. It is noted from various measurements, e.g., [18], [19], [20], that the propagation loss difference depends on the frequency ratio as follows:

$$
\Delta L_{F}=L_{F}\left(f_{1}\right)-L_{F}\left(f_{2}\right)=k \log \left(\frac{f_{1}}{f_{2}}\right) d
$$

Where $L_{F}\left(f_{1}\right)$ and $L_{F}\left(f_{2}\right)$ are the propagation losses at frequencies $\mathrm{f}_{1}$ and $\mathrm{f}_{2}$ in $\mathrm{MHz}, \mathrm{k}$ is a constant to be determined, and $\mathrm{d}$ is the distance through the foliage in meters. To find the optimum value of $\mathrm{k}$, consider the squared errors:

$$
S E=\sum_{i=1}^{N}\left(\Delta M_{i}-k \log \left(\frac{f_{1}}{f_{2}}\right) d_{i}\right)^{2}
$$

Here $\Delta M_{i}$ represents the difference between measured losses at $f_{1}$ and $f_{2}$ at point $i$. Differentiating this equation with respect to $\mathrm{k}$ and equating the result to zero, gives:

$$
k=\frac{\left[\sum_{i=1}^{N} \Delta M_{i}\right]}{\left[\log \left(\frac{f_{1}}{f_{2}}\right) \sum_{i=1}^{N} d_{i}\right]}
$$

Using different sets of measured data, it is found here that $\mathrm{k}=0.1306$, also note that $\mathrm{f}_{1}$ is $300 \mathrm{MHz}$. After combining (16) and (17), the propagation loss with dry foliage is expressed as:

$$
\begin{aligned}
& L_{F}=3.12+45.11 \log (d)+0.1306 \log (f) d- \\
& 0.324 d
\end{aligned}
$$

\section{Results}

The loss calculated by equation (20) is compared with measured values [18] in Fig. 2 below. The figure reveals excellent agreement between measurements and the optimized model predictions at $300 \mathrm{MHz}$ as well as 1900 MHz.

After rainstorms, the trees and other components of the forest become wet. This affects the attenuation of the wave passing through vegetation areas. Following the general lines of the optimization procedure described above, the excess attenuation due to wet state of the vegetation $L_{\mathrm{EW}}$ may be represented by:

$$
L_{E W}=0.00071 f^{0.81} d^{0.95}
$$

$\mathrm{f}$ is in $\mathrm{MHz}$ and $\mathrm{d}$ in meters. Equation (21) is verified by comparison with measured data, [18]- [19], as Fig. 3 shows. 


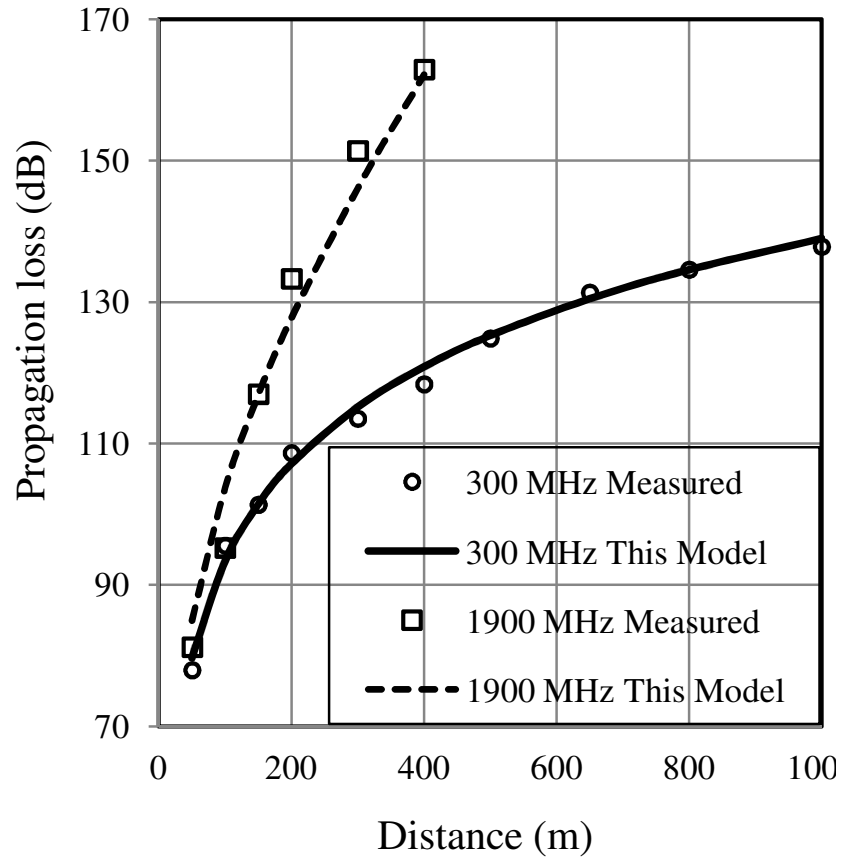

Fig. 2. Propagation loss vs. distance inside dry foliage for different frequencies, where measured data are compared with the proposed model predictions.

$$
\begin{array}{cc}
L_{W F}=3.12+45.11 \log (d)+0.1306 \log (f) d- \\
0.324 d+0.00071 f^{0.81} d^{0.95}
\end{array}
$$

The validity of the model for wet foliage is demonstrated in Fig. 4 where measurements again agree with predictions.

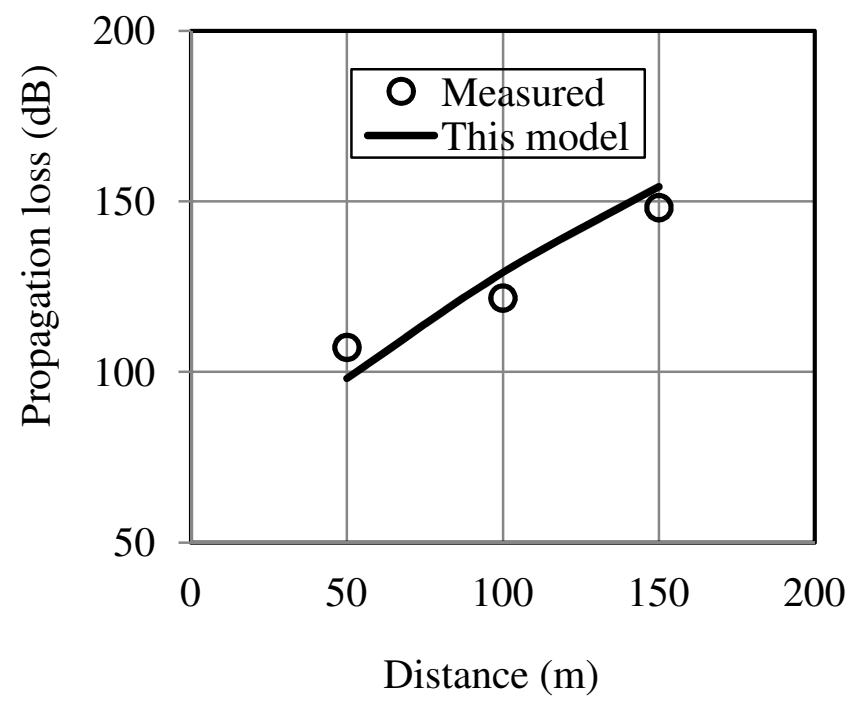

Fig. 4. Measured and calculated propagation loss with wet foliage vs. distance at $1900 \mathrm{MHz}$.

Accordingly, a comprehensive equation is given here that involves various propagation loss parameters including the distance through the vegetation, operating frequency, and wet/dry foliage states. It is worth noting here that equation (20) pertains to dry woodland area whereas (22) can be used for wet woodland area, e.g., after rainfall/snowfall.

\section{Conclusions}

This paper offers a new optimized model for propagation in woodland environments. The method of least squares is used to obtain optimum parameters for the model. Different components affecting the propagation loss are taken into consideration including the distance through foliage, frequency of operation, and wet/dry states of the trees after rainfall/snowfall. The results presented in this paper show that the excess fading due to moist state of the vegetation has significant impact on the signal level. The validity and usefulness of the model is shown through comparison with measurements where excellent agreement can be observed.
Augmenting (21) to (20), the proposed model for wet foliage becomes:
Fig. 3. Excess attenuation coefficient in $\mathrm{dB} / \mathrm{m}$, of wet over dry foliage vs. frequency. 


\section{Acknowledgement}

The author would like to thank Jordan University of science \& Technology for the financial support to participate in this conference.

\section{References}

[1] Joel Iloke, "Suitability of the Early ITU Model in Parts of Cross River State Nigeria," International Journal of Scientific Engineering and Applied Science (IJSEAS) - Volume-4, Issue-9, September 2018

[2] A. S. Adegoke, David Siddle, S. O. Salami, "Vegetation attenuation and its dependence on foliage density," European Journal of Engineering and Technology, Vol. 4, No. 3, pp. 1-7, 2016.

[3] Theodor Tamir, "On radio-wave propagation in forest environments," IEEE Transactions on Antennas and Propagation, Vol. 15 , Issue 6, pp. 806-817, Nov. 1967.

[4] M. L. Meeks, "VHF propagation over hilly, forested terrain," IEEE Transactions on Antennas and Propagation, Vol. 31, Issue 3, pp. 483-489, May 1983.

[5] Karl Low, "UHF measurement of seasonal fieldstrength variations in forests," IEEE Transactions on Vehicular Technology, Vol. 37, Issue 3, pp. 121 124, Aug 1988.

[6] R. K. Tewari, S. Swarup, and M. N. Roy, "Radio wave propagation through rain forests of India," IEEE Transactions on Antennas and Propagation, Vol. 38, Issue 4, pp. 433-449, Apr. 1990.

[7] M. O. Al-Nuaimi and A. M. Hammoudeh, "Measurements and predictions of attenuation and scatter of microwave signals by trees," IEE Proceedings.-Microwaves, Antennas \& Propagation, Vol. 141, No. 2, pp. 70-76, April 1994.

[8] J. C. R. Dal Bello, G. L. Siqueira, and H. L. Bertoni, "Theoretical analysis and measurement results of vegetation effects on path loss for mobile cellular communication systems," IEEE Transactions on Vehicular Technology, Vol. 49, Issue 4, pp. 12851293, Jul. 2000.

[9] M. H. Hashim and S. Stavrou, "Measurements and modelling of wind influence on radiowave propagation through vegetation," IEEE Transactions on Wireless Communications, Vol. 5, Issue 5, pp. 1055-1064, May 2006.

[10] Yu Song Meng, Yee Hui Lee, and Boon Chong Ng, "The effects of tropical weather on Radio-wave propagation over foliage channel," IEEE Transactions on Vehicular Technology, Vol. 58, No. 8, pp. 4023- 4030, Oct. 2009.

[11] M. S. H. Al Salameh, and M. M. Qasaymeh, "Effects of Buildings and Trees on Satellite Mobile Communications," International Journal of Electronics, Vol. 91, pp. 611-623, 2004.

[12] Yu Song Meng, Yee Hui Lee, and Boon Chong Ng, "Empirical near ground path loss modeling in a forest at VHF and UHF bands," IEEE Transactions on Antennas and Propagation, Vol. 57, No. 5, pp. 1461- 1468, May 2009.

[13] M. S. H. Al Salameh, "Vegetation attenuation combined with propagation models versus path loss measurements in forest areas," World Symposium on Web Application and Networking- International Conference on Network Technologies and Communication Systems, March 22-24, 2014, pp. 120-124, Hammamet, Tunisia.

[14] Sogo Okamura and Tomohiro Oguchi, "Electromagnetic wave propagation in rain and polarization effects," Proceedings of the Japan Academy, Series B, Vol. 86, 2010.

[15] M. S. H. Al Salameh, "Waves and Fields of Wireless Communications and Electricity: HealthEffects and Unconventional Utilizations," Lap Publishing, printed in USA and in the UK, 2011.

[16] S. R. Saunder, and A. Aragon-Zavala, "Antennas and Propagation for Wireless Communication Systems," $2^{\text {nd }}$ Edition, John Wiley \& Sons, Chichester, England, 2007.

[17] Y. S. Meng, Y. H. Lee, and B. C. Ng, "Study of propagation loss prediction in forest environment," Progress In Electromagnetics Research B, Vol. 17, 117-133, 2009.

[18] G.G. Joshi, C.B. Dietrich Jr., C.R. Anderson, W.G. Newhall, W.A. Davis, J. Isaacs and G. Barnett, "Near-ground channel measurements over line-ofsight and forested paths," IEE Proc.-Microw. Antennas Propag., Vol. 152, No. 6, December 2005.

[19] N. C. Currie, E. E. Martin, F. B. Dyer, "Radar Foliage Penetration measurements at Millimeter Wavelengths," ESS/GIT-A-1485-TR-4, Georgia, Atlanta GA, 31 Dec 1975.

[20] M. O. Al-Nuaimi and R. B. L. Stephens, "Measurements and prediction model optimization for signal attenuation in vegetation media at centimetre wave frequencies," IEE Proc. Microw. Antennas Propag., Vol. 145, No. 3, pp. 201-206, June 1998. 